\title{
Silver diamine fluoride as a minimally invasive and aerosol free approach in pediatric dentistry: An extensive case series
}

\author{
Abhishek Soni ${ }^{1}$, (1) Sanchit Paul ${ }^{2}$, (D) Priyanka Sachdeva ${ }^{3}$
}

\section{Highlights}

This study reports usage of SDF as extensive case series in various form of SMART, in proximal deep caries as indirect pulp therapy medicament, sealants and in MIH in various clinical situations in pediatric dentistry.

${ }^{1}$ Chief Pediatric Dentist, V anilla Smiles, Soni Capital 1237 Apte Rd.,Shivajinagar Pune, Maharashtra, India

${ }^{2}$ Chief Pediatric Dentist, Tooth Tales, House

No. D-2, Sec-P-3,Greater Noida, U.P., India

3 Assistant Professor, Department of Pediatric and Preventive Dentistry, School of Dental Sciences, Sharda University, Knowledge Park 3, Greater Noida, U.P., India

\section{Correspondence:}

Tooth Tales, House No. D-2, Sec-P-

3, Greater Noida, U.P., India

E-mail address:

dr.sanchit@gmail.com
With correct diagnosis and appropriate treatment planning, SDF can be an indispensable tool for both minimally invasive pediatric dentistry (MIPD) and aerosol free dentistry (AFD) especially in pandemic times.
SDF parental handout form, clinical protocol along with decision making tree and usage of teflon tape could be immensely helpful for practitioners for administering SDF in both primary and permanent dentition routinely.

\section{Abstract}

The current pandemic of COVID-19 warrants a repeal from conventional dentistry to an aerosol free, minimally invasive yet maximally effective clinical approach. Silver diamine fluoride (SDF) is an established modality for caries arrest in children fulfilling all the above. This extensive 25 case series highlights various clinical situations in which SDF was successfully used in children between 1-12 years of age with asymptomatic carious lesions of ICDAS score 2 or more in primary and permanent teeth. Primary outcome measure was caries arrest in the form of the hard and shiny lesion with no/minimal sensitivity. The secondary outcome measure was the zombie effect which was the residual staining on other tooth surfaces and indications of substantivity. SDF can be effectively and efficaciously used across different behaviour patterns and age groups of children in a various clinical scenario for both primary and permanent teeth routinely experienced in a paediatric dental office especially during these pandemic times with minimal aerosol generation.

Keywords: Case Reports; COVID-19; Dental Caries; Dental Enamel Hypoplasia; Fluorides; Silver Diamine Fluoride 


\section{INTRODUCTION}

The current crisis of COVID-19 caused by the pathogen SARS-CoV-2 has brought a significant impact on the dental profession as a whole. Apart from the current pandemic, dental care setups have always been at a risk for spread of diseases, that can be transmitted through droplets. ${ }^{1}$ The aerosols that are generated due to the use of handpieces, ultrasonic scalers and three - way syringe expose both the dental team and patients to a higher and consistent risk.

Recently, recommendations have been published $^{2}$, which emphasizes the usage of minimally invasive procedures eliminating aerosol generation largely in children. The need of the hour warrants an aerosol free yet cost effective approach for management of dental caries. In this regard, we would like to highlight the use of silver diamine fluoride (SDF), the use of which as a safe and effective therapy to treat and prevent caries is already well established. ${ }^{3}$

Silver's antimicrobial effects in conjunction with fluoride ion's remineralizing effects make SDF a safe, inexpensive and efficacious modality which is simple to implement in various dental settings while targeting children and those with special health care needs (SHCN). ${ }^{4}$ Thus, SDF is an integral tool for providing minimally invasive paediatric dentistry (MIPD) coupled with aerosol free dentistry (AFD).

Although we have numerous studies including a recent systematic review and meta-analysis on clinical effectiveness of SDF-9, this extensive clinical case series of 25 cases is to emphasize how SDF can be effectively utilized in a several clinical situations in dental office for both primary and permanent teeth and its applicability suiting the current pandemic.

\section{CASE REPORT}

The present case series is written in accordance with PROCESS guidelines for case series reporting. ${ }^{10}$ All cases discussed in this current case series are multicentric; retrospective in design and consecutive in nature. Children with age range between 1-12 years with asymptomatic carious lesions of ICCMS/ICDAS ${ }^{11,12}$ score 2 or more (without pulpal involvement-clinically and radiographically) were recruited from February 2018 and followed up till May 2020. Permanent teeth with either smooth surface demineralization (ICDAS 2) or affected by molar incisor hypomineralization (MIH) with/without sensitivity/defect were also included. Any carious lesion which had signs and symptoms of irreversible pulpitis - clinically or radiographically, or parents not consenting to SDF and without complete documentation was excluded. Informed consent was taken from parents and/or guardians.

Parents/guardians were informed and educated about SDF using a dedicated presentation through our respective web portals and clinical handouts; its facts, frequency of application and its role in the prevention and management of caries, along with the probable risks and alternatives reported in the literature in the form of UCSF consent form. ${ }^{13} \mathrm{An}$ information handout in simple non clinical terms was given to each parent before commencement of the procedure. All cases followed the SDF application protocol for occlusal or proximal lesion of primary or permanent teeth as described by Crystal and Niederman ${ }^{4}$ (Figure 1). A pediatric dental practice - based clinical decision tree was made to determine the best clinical outcome for the patient (Figure 2). All cases are categorized as per the clinical situations which are routinely experienced in a pediatric dental office.

Frankl's behavior rating (FBR) scale was used to determine the child's behavior and had a significant impact on the choice of treatment and the treatment modality rendered. Pre- cooperative children were treated either under Nitrous Oxide Oxygen Inhalation Sedation (NO-OIS) if they could accept nasal hood/mask or in knee to knee/lap position when they couldn't accept mask or there was no parental consent for sedation. 
Informed and written consent (UCSF protocol) after due audio-visual counselling

Universal precautions \& armamentarium preparation

(goggles, tissue papers, plastic dapen dish/microsponge/petroleum jelly/SDF)

Patient preparation - 6 or 8 handed dentistry with/without physical restraint/NO-OIS

Isolation of the operating field (cotton roll/rubber dam) after petroleum jelly on the circumoral area

In case of cavitation:

Selective caries removal from the walls of lesion
In case of smooth surface:

Removal of biofilm/debris

with 38-39 sharp spoon excavator/slow speed handpiece and round carbide bur

Light drying (with air/cotton rolls/guaze) of the affected tooth surface.

Use of rubber dam is preferred if the child can cooperate

Application of SDF directly to the affected tooth surface with help of micro sponge brush (removal of excess by dabbing before application).

Use of Superfloss for proximal lesions

Drying with a gentle flow of compressed air for at least 1 minute if possible

Removal of excess SDF with guaze/cotton

Isolating the field for further 3 minutes (whenever possible)

Application of Topical Fluoride Varnish (Smooth Surface)

High strength GIC (Type IX or Equia Forte/SSC for SMART)

Post-operative instructions as per protocol

Motivational counselling for diet and home oral hygiene measures including amount of fluoride toothpaste and brushing technique

Figure 1. Silver diamine fluoride clinical protocol followed (Adapted by Crystal and Niederman, 20194) 


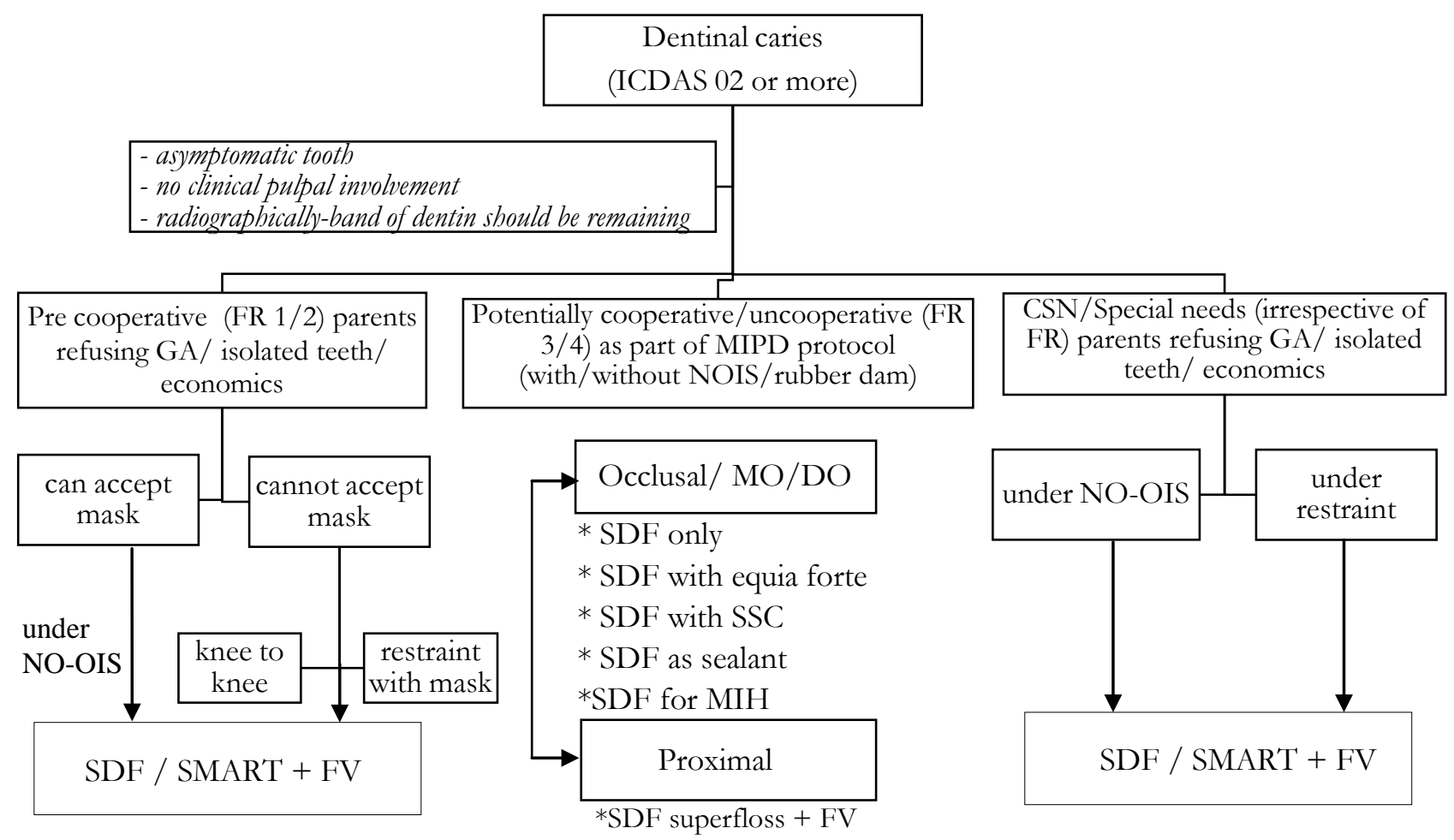

ICDAS: International Caries Detection and Assessment System; FR: Frankl Rating; CSN: Child with Special Needs; GA: General Anesthesia; NOOIS: Nitrous oxide- Oxygen Inhalational Sedation; MO: Mesio-Occlusal; DO: Disto- Occlusal; SDF-: Silver Diamine Fluoride; SMART: Silver Modified Atraumatic Restorative Treatment; FV: Fluoride Varnish; SSC: Stainless Steel Crown

Figure 2. Silver diamine fluoride clinical decision-making tree

Children with SHCN were treated with physical restraints with oral sedation or NO-OIS or combination of both.

For cooperative/potentially cooperative children, selective caries removal was done from the walls of the cavitation utilizing the aerosol free approach and silver modified atraumatic restorative treatment (SMART) was employed for occlusal caries as per MIPD protocol. The choice of SDF used for all cases was 38\% Advantage Arrest $^{\mathrm{TM}}$ (Elevate Oral Care, West Palm Beach, FL), High strength glass ionomer cement (GIC) used was either Type IX GIC or EQUIA Forte (GC America). For SMART with Stainless Steel Crowns (SSC), either 3M crowns (ESPE,St. Paul, Minnesota, USA) or Kids crowns (Shinghung, Seoul, Korea)were used with halls/conventional technique. For the inter proximal lesions, SDF was applied with the use of a micro brush and Super Floss ${ }^{\circledR}$ (Oral-B, The Procter \& Gamble Company, Cincinnati, $\mathrm{OH})$.

Intra operative variation was minimized as the application was done by experienced, exclusive pediatric dentists who followed the same adapted protocol by Crystal and Niederman ${ }^{4}$ (Figure 1). This was further reduced by independent observation by third author who agreed with all inclusion and exclusion criteria for this case series.

\section{Case series descriptives and outcome measures}

A total of 25 cases as per inclusion criteria were included in this case series of SDF in different clinical situations. The teeth were scored using the International Caries Classification and Management System (ICCMSTM) with ICDAS for both clinical severity and activity. ${ }^{7}$ Baseline data of feeding history, diet diary and oral hygiene practices were recorded in form of E-health records for each case.

All patients received biannual SDF application along with 3 monthly fluoride varnish (FV) application over the lesion. ${ }^{14}$ Intensive SDF regimen in form of 2-3 applications within a month was followed in treatment for MIH and severe early childhood caries (SECC). In case of no/minimal biofilm accumulation after a year, FV 
was limited to 6 months along with SDF biannual applications barring SMART restorations. At post-operative and 3 months follow up visits, DSLR camera photography if possible, as per child cooperation or a minimum of Mobile Dental photography (MDP) was done to ensure clinical documentation. Radiographs were taken if indicated at 6 months interval to assess the stability in approximal lesions or tertiary dentin formation in occlusal lesions.

Primary outcome measure was caries arrest in form of hard and shiny lesion with no/minimal sensitivity. Secondary outcome measure was the zombie effect which was the residual staining on other tooth surfaces and indication of long term substantivity. Parents were also motivationally interviewed for ongoing home oral hygiene measures and diet modification including cessation of night time feeding practices. A note was made about parents' acceptability towards MIPD approach and black staining. In case of any adverse event like intolerable pain, acute periapical abscess or extraoral facial cellulitis, the patient was immediately attended to. Subsequent treatment in form of extraction followed by space maintainer if indicated was duly carried out.

\section{DISCUSSION}

The dental profession is currently faced with a challenging task in the wake of COVID-19 which has a respiratory route of transmission through droplets and/or aerosols. Management of dental carious lesions has seen a metamorphosis from the traditional surgical approach towards a biological approach. MIPD is the need of the hour to close the gap between the evidence base and clinical pediatric dental practice. The characteristics of MIPD are the delivery of effective, risk based, patient centred caries care that controls initial caries non-operatively whilst conserving tooth structure at all times.

The authors chose the cases for MIPD with SDF based on clinical presentation of different cases. All children universally presented with extended breast-feeding practices, nocturnal bottle-feeding habit, limited oral hygiene post feeding in pre-cooperative age group. Most of them either had not started brushing or inadequacy in form of non-usage of fluoride toothpaste and/or improper technique was evident. For older age group, the diet was primarily cariogenic with frequent in-between meals sugar exposure. The role of home care was reinforced before undertaking any treatment through motivational counselling at first visit only. They were given a folder with all necessary education and information in the form of SDF handout and consent form and were encouraged to go through ECC and SDF/SMART and other MIPD approaches on the authors' website, social media channels for recorded videos and other authenticated reading resources like AAPD website and scientific literature. Parental expectation and education is an essential step before undertaking any SDF procedure as fully motivated and informed parents minimize SECC activity.

Rapid adoption of SDF despite the nonaesthetic result indicates its preference against the discomfort caused by traditional operative dentistry and invasive pharmacological approaches, which are supported by various surveys on parent choices regarding SDF. 15 However, the cost effectiveness of SDF may also be a contributing factor. As highlighted in one of the cases of pre-cooperative child case series, active lesions were arrested with biannual applications of SDF at 12 months follow up avoiding hospitalization and instilling a positive dental attitude for the 30 months old precooperative child (Figure 3). 


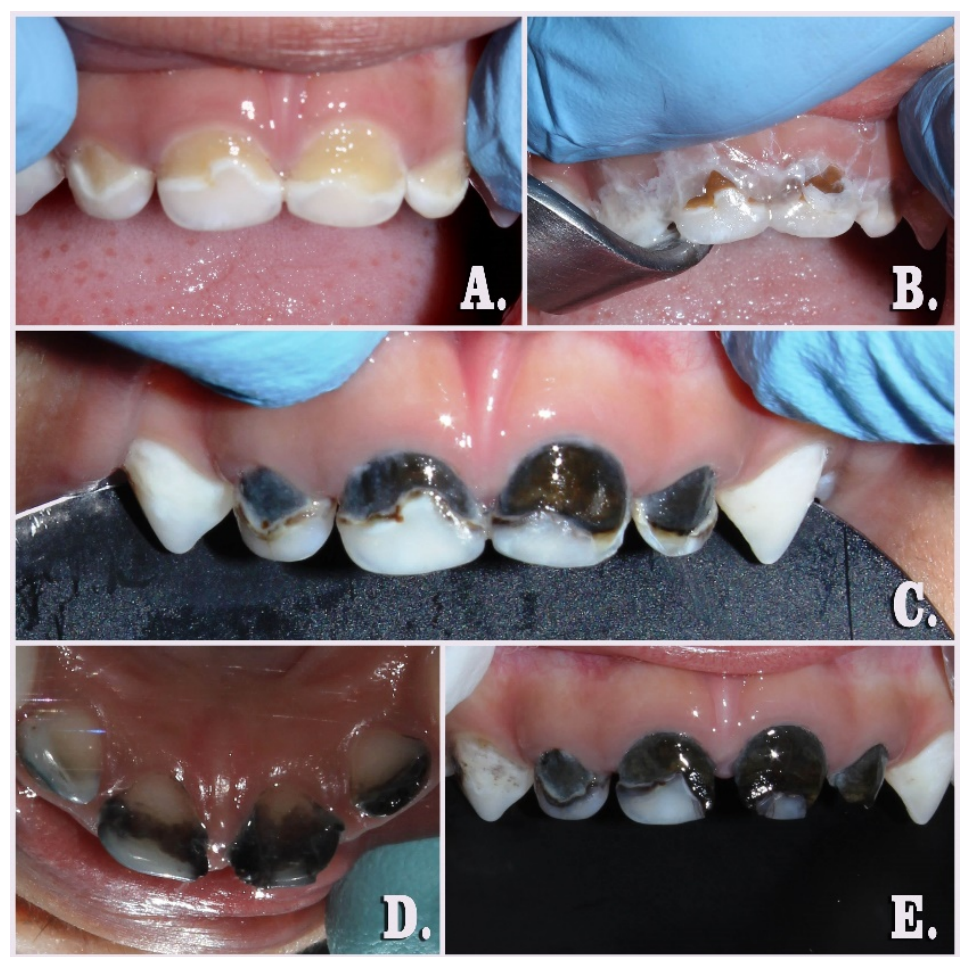

Figure 3. SDF in precooperative child. A: Pre-operative, B: Immediate post op followed by FV, C: 3 months followup, D: 6 months follow-up and second application, E: 12 months follow up and zombie effect seen with 53 and 63

As depicted in Figure 4, the use of SDF is not only limited to pre-cooperative children but also serves as an effective MIPD approach across different age groups of children. For children between 2 to 4 years of age, the same position was utilized for SDF application and treatment was done under NO-OIS provided the child was able to accept the nasal hood. Authors strongly believe that NO-OIS even under restraint coupled with MIPD like SDF/SMART instils positive attitude for subsequent visits, especially for SCHN. For grown up children (preschoolers, >5) /FR3/4, MIPD was done mainly under rubber dam under NO-OIS. Apart from primary teeth, even permanent teeth especially affected by MIH were part of this decision making.

To the best of our knowledge, this is the first study highlighting a clinical decision-making tree for SDF in pediatric dental office, while using ICCMS tool to manage active carious lesions. ICCMS $^{\mathrm{TM}}$ criteria are based on three important pillars of critical analyses, research, and clinical feedback on the best alternate approaches to move away from the restorative care cycle towards more of prevention-based system.

The protocol followed for SDF was biannual application with a recurring three month follow up coupled with fluoride varnish (FV). Studies have highlighted that biannual clinical application is almost thrice more effective than applying annually only. ${ }^{16,17}$ Using FV in conjunction with SDF has many added benefits like masking of unpleasant taste of amine of SDF, minimizing washing away of SDF with saliva, increase or maintain SDF efficacy and keep the caries activity to minimal for first year after SDF therapy. ${ }^{14}$ Care should be taken regarding dosing in young children and separate brush for varnish over SDF tooth as it might lead to inadvertent spill and zombie effect. ${ }^{18}$ 


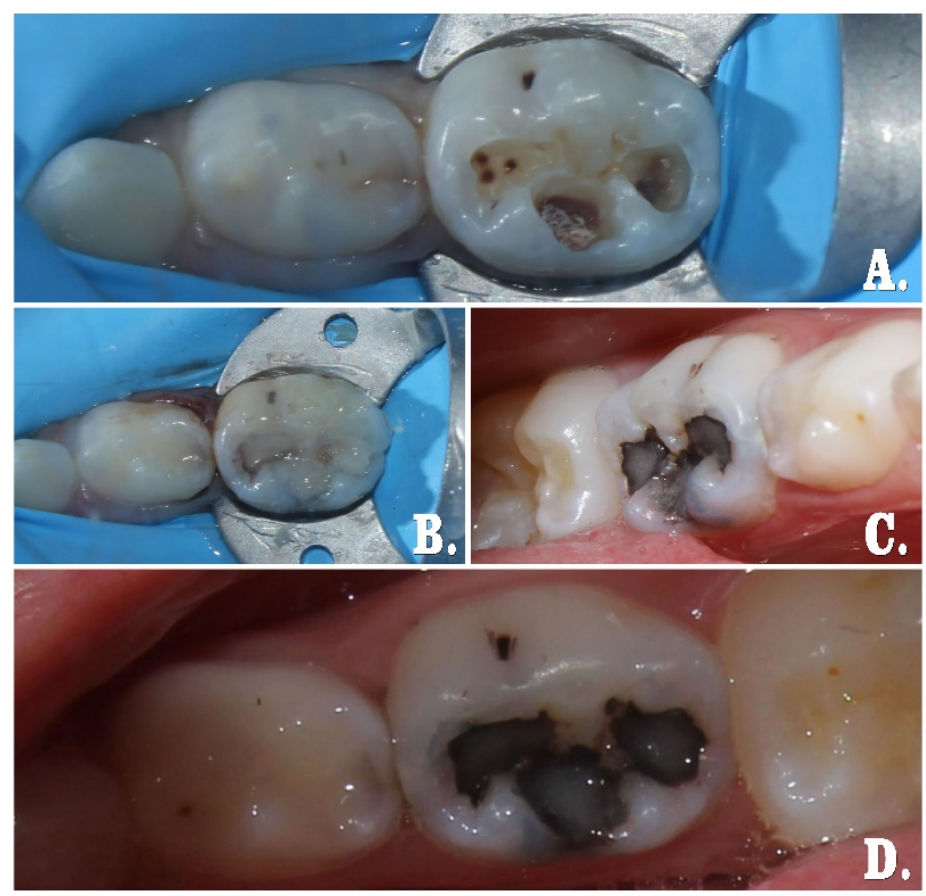

Figure 4. SDF as SMART with MIPD approach. A: Pre-operative, B: Immediate post op followed, C: 6 months follow-up, D: 12 months follow up

\section{SMART (Silver Modified Atraumatic} Restorative Treatment/therapy) is a most recent, non-invasive and inexpensive ART of this millenium. ${ }^{19}$ The amalgamation of glass ionomers hydrophilicity, bio-chemical property, fluoride release, quick set, ease of handling along with cariostatic property of SDF makes it literally the smart way of doing restorations in a precooperative or a child with limited coping ability. We achieved clinical and radiographic success when using SDF under SMART restoration (Figure 5) and under Hall's crown, which is again an established clinical procedure in MIPD protocol in accordance with ICCMS management protocol. SDF coupled with Hall's crown has an added advantage of a better coronal seal as compared to the conventional SMART glass ionomer restoration in high caries risk children as was seen in one of our cases (Figure 5).

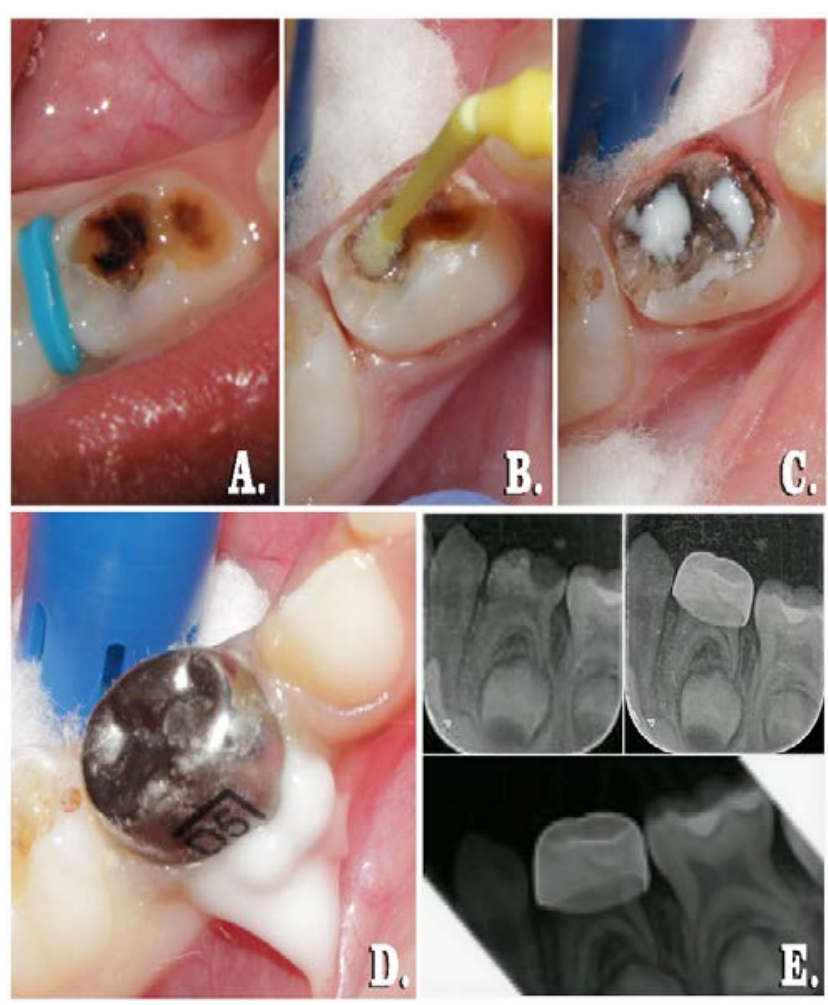

Figure 5. SDF with SMART HALLS crown. A: Preoperative with elastics placed, B: Intraoperative, C: Immediately after application of SDF, D: Post stainless steel crown cementation, E: Radiographs at Preop, follow up at 12 months and 24 months 
Conventional treatment approach of proximal carious lesions initiates a vicious cycle of retreatment which eventually leads to more structural loss. ${ }^{20}$ Large area of contact coupled with limited salivary access promotes a more acidogenic environment which is conducive to the development of caries in approximal surfaces of primary teeth. ${ }^{21}$ Using an expanding woven floss saturated with $38 \%$ SDF is the mainstay of nonoperative/ restorative caries care for these lesions. ${ }^{22}$
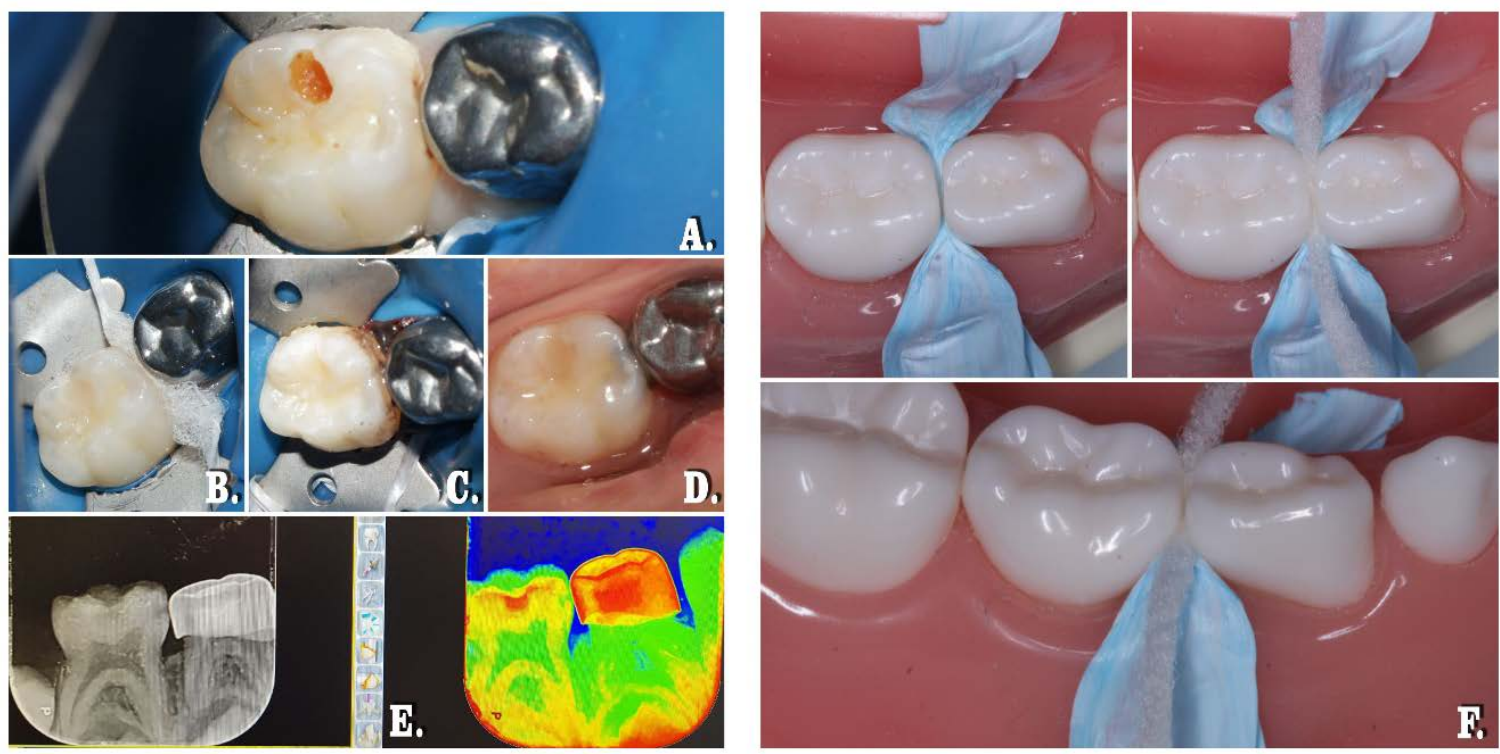

Figure 6. SDF in approximal lesion. A: Pre-operative, B: Intraoperative - Expanding floss saturated with 38\% SDF, C: Immediate post-operative - SDF and tattoo of the interproximal papilla, D: 12 months clinical follow-up. Notice the stability of the lesion clinically, E: 12 months radiographic follow-up. Notice the stability of the lesion radiographically; F: Use of Teflon tape to protect the interdental papilla

The mandibular primary incisors are the most resistant to decay owing to the remineralizing effect of the saliva and self-cleansing action of the tongue. Presence of incipient lesions in the mandibular incisors present a unique challenge owing to the size of the tooth, moisture control, closed dentition and lack of retentive form. SDF penetrates enamel to a depth of upto 50 microns, and approximately 2-3 times more fluoride is retained than that delivered by fluoride varnish alone, improving the surface resistance to further progression. ${ }^{4}$ We used the similar approach for a 26-month girl (Figure 7) so to avoid invasive drilling but also arrest caries progression and we had a stable result for 6 months (the patient is
In our study initial active proximal lesions ICCMS RA2;3, RB4,5were managed using 38\% SDF (Figure 6A,E). Similarly, SDF is an excellent tool for ICDAS 4/5 lesion with still intact remaining dentin. It can be used to arrest the caries progression and can be used along with any bioactive material to remineralize and have hermitic seal with SSC. 


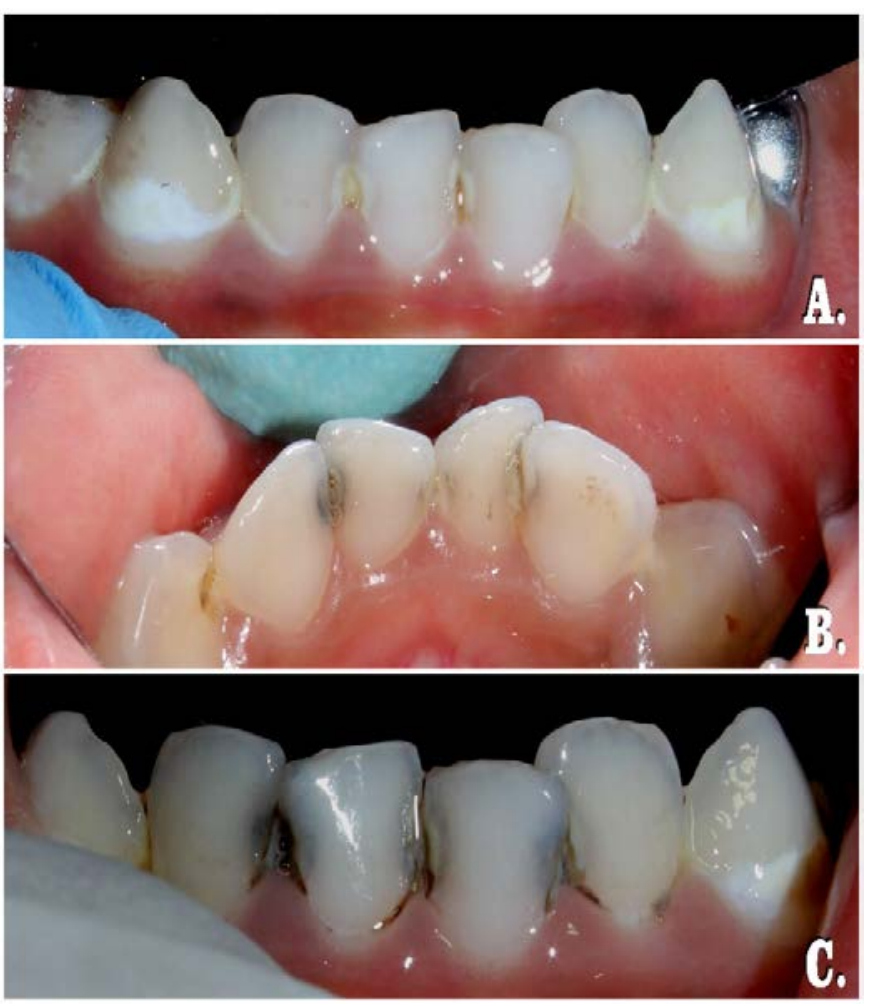

Figure 7. SDF in mandibular anteriors. A: Preoperative; B: 6 months follow-up, C: 12 months clinical follow-up

In this study, SDF has shown promising results in terms of caries arrest and lesion stabilization in permanent teeth (affected by $\mathrm{MIH})$. The data for MIH affected teeth in the literature is limited. ${ }^{25}$ Authors tried extending the usage even as sealants in permanent molar pits and fissures (Figure 9A,B); while having achieved stable results in primary molars (Figure 9C,D). The rationale again is similar to that these teeth have heightened sensitivity to even regular sealant procedure which requires etching and air-drying process. SDF not only eliminates that step but also provides substantivity in terms of remineralisation and subsequent sealing. Stable result was achieved in terms of hard and shiny lesion with no sensitivity in molar teeth affected by MIH as judged at 6-month follow-up (Figure 10). Although, long term follow-up is needed to validate these results, we strongly believe that SDF has and will become a mainstay treatment modality for sensitivity and caries arrest in such situations.
MIPD approaches not only reduce the need for recurrent sedation or GA but also improve the overall behaviour and experience of children with SHCN. In one of the cases where 2.5-year-old girl (Figure 6C,D) who was preterm and extremely low on birth weight and haemoglobin; SDF in these cases is our best bet to not only delay invasive treatment but minimize further caries activity. In our clinical experience, parents of these children were significantly more accepting to alternative treatment approaches that could prevent or delay GA. Some of the children eventually never needed general anaesthesia as along with parental motivation, they were able to stabilize their treatment needs and then accepted treatment chair side along with NO-OIS or oral sedation.

SDF has huge safety margin if proper dosing of 1 drop per $10 \mathrm{~kg}$ per visit is followed ${ }^{26}$. But it has some limitations in form of suspected adverse events or reactions. Most primary and common is black discolouration or staining or white patch of oral mucosa if SDF accidentally comes in contact. ${ }^{7}$ Although despite this, it has been accepted highly by parents especially as an excellent alternative to other invasive pharmacological approach in young age group ${ }^{27}$ Also, misdiagnosed pulpal status can lead to continuation of formation of dento-alveolar abscess in long term. The authors wish to highlight here that SDF has minimal chances to induce pulpal inflammation as already highlighted by Rossi et al recently. ${ }^{28}$ As already discussed, metallic taste of the amine can be easily masked by superimposing flavoured fluoride varnish or simple petroleum jelly. The dental offices also received phone calls from parents showing concerns with tattooing observed in the interdental papilla. The authors recommend application of petroleum jelly using an applicator tip or the use of Teflon tape onto the interdental papilla before application of SDF interproximally (Figure 6F). 

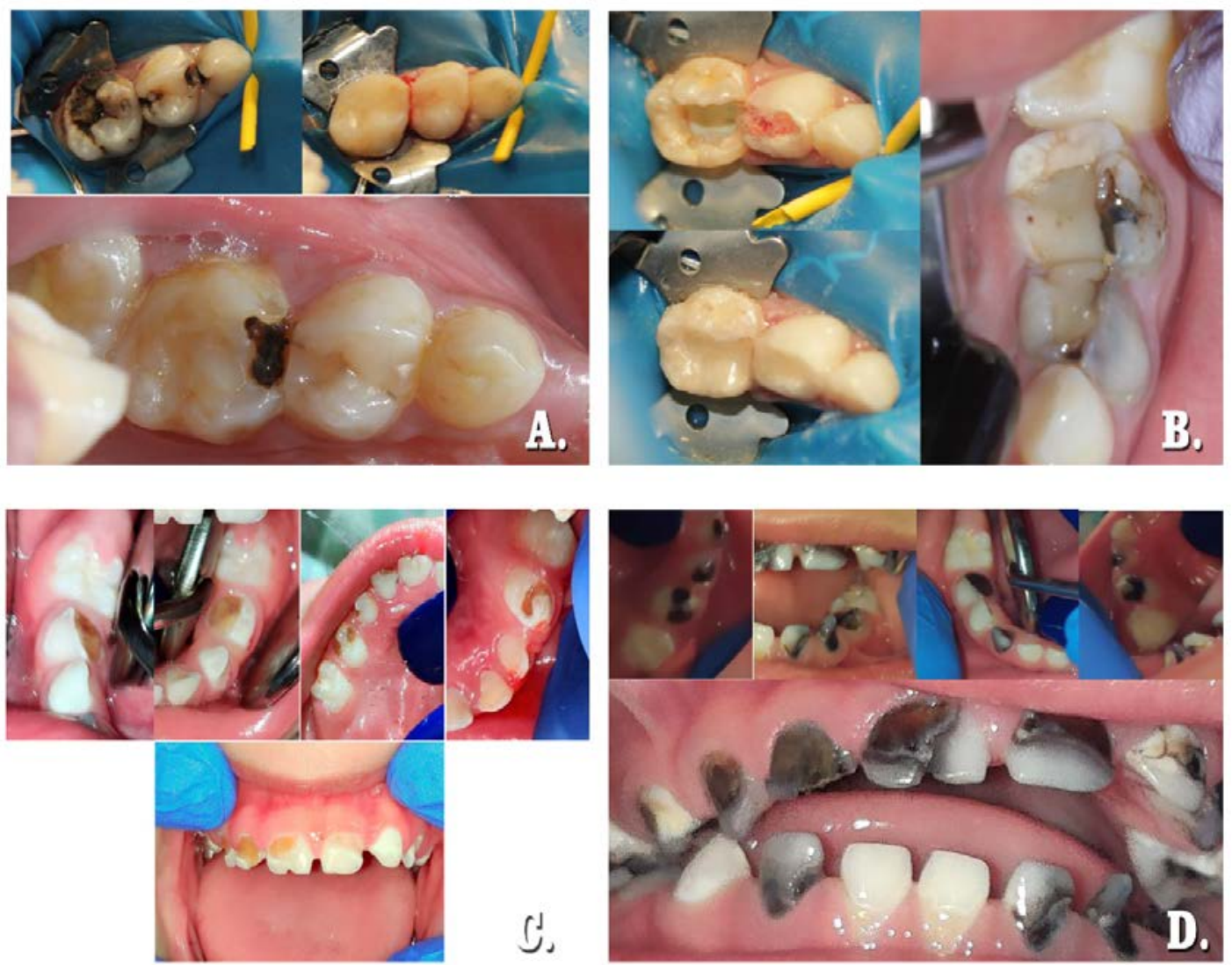

Figure 8. SDF to arrest secondary caries. A: proximal, B: Occlusal, C: SDF in children with special needs: Preoperative, D: SDF in children with special needs: 6 months follow-up

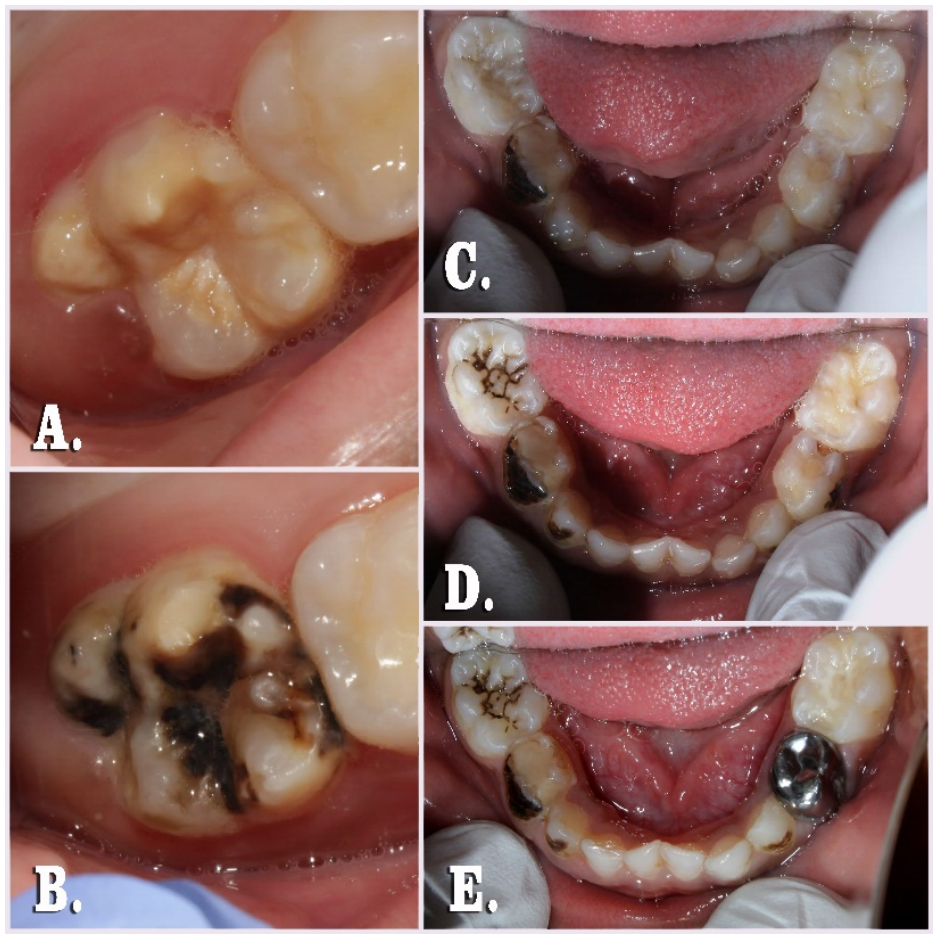

Figure 9. SDF as sealant in MIH affected permanent first molar. A: Preoperative, B:Postoperative, C: SDF as sealant in primary molar i.r.t. 85 - Pre operative, D: SDF as sealant in primary molar i.r.t. 85-12 months follow-up, E: SDF as sealant in primary molar i.r.t. 85-24 months follow-up 

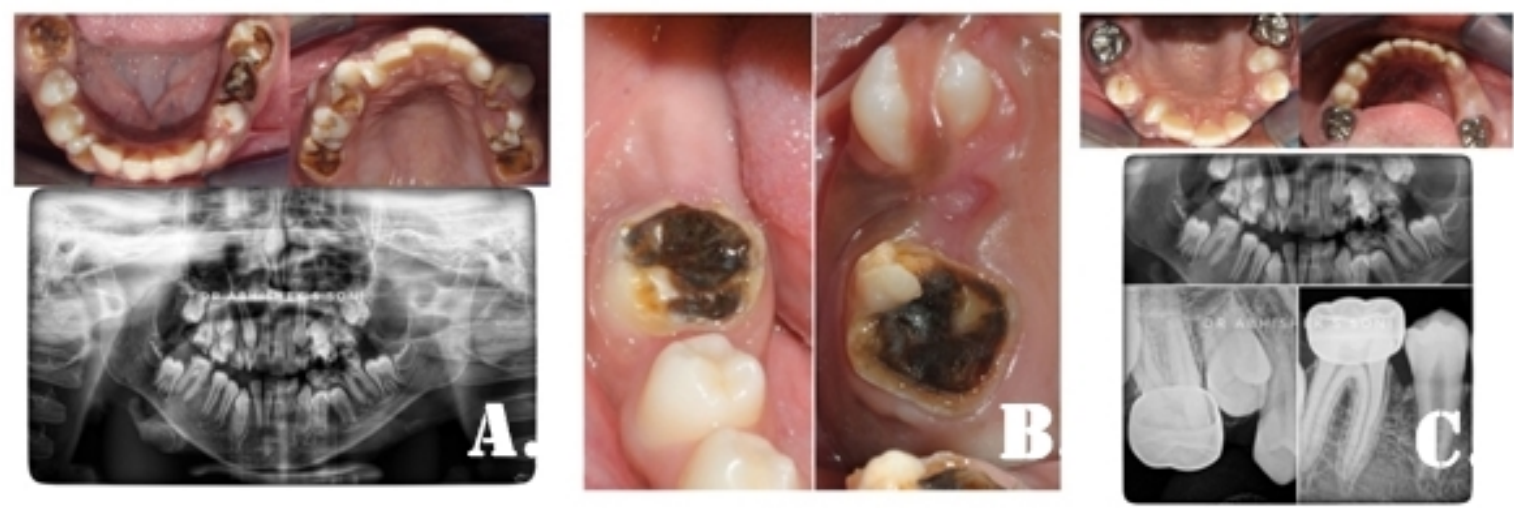

Figure 10. SDF as IPC agent in MIH affected permanent molars. A: Preoperative, B: Intraoperative, C: Postoperative

Case reports have a low ranking in the evidence hierarchy. However, the authors aim to present their clinical experience and idiographic emphasis on the use of SDF through a multicentric extensive case series in the current COVID-19 times. However, heterogeneity in clinical practice among centres may have affected the choice of management strategy and the treatment rendered. The duration of follow up is another important criterion. The case series is retrospective in nature which does not establish a cause-effect relationship. A case control study would have provided significant results and the authors aim to undertake the same in future. Future research of SDF on MIH and permanent teeth as sealants in given cases, SCHN along with novel approaches like Nano Silver Fluoride and mixing of SDF plus Povidone Iodine needs to be researched.

\section{CONCLUSIONS}

The multi-faceted use of SDF coupled with ease of application whilst requiring minimum equipment for the operator and as an aerosol free and painless treatment option for the child makes it a cardinal tool for pediatric dentists especially during COVID-19 pandemic. This paper aims to highlight the innovative parental handouts, clinical decision-making tree along with clinical protocol to determine the best evidence-based intervention for the use of SDF in a dental office. With all the minimally invasive procedures available at the clinician's disposal today, it is not wrong to presume that SDF is the crux on which the minimally invasive paediatric dentistry and aerosol free dentistry lies.

\section{REFERENCES}

1. Peng X, Xu X, Li Y, Cheng L, Zhou X, Ren B. Transmission routes of 2019-nCoV and controls in dental practice. Int J Oral Sci 2020;12:1-6

2. Mallineni SK, Innes NP, Raggio DP, Araujo MP, Robertson MD, Jayaraman J. Coronavirus disease (COVID-19): Characteristics in children and considerations for dentists providing their care. Int J Paediatr Dent 2020;30:245-250

3. Slayton RL, Urquhart O, Araujo MWB, Fontana M, Guzmán-Armstrong S, Nascimento MM et al. Evidence-based clinical practice guideline on nonrestorative treatments for carious lesions: A report from the American Dental Association. J Am Dent Assoc. 2018;149:837-849

4. Crystal YO, Niederman R. Evidence-Based Dentistry Update on Silver Diamine Fluoride. Dent Clin North Am 2019; 63:45-68

5. Jiang M, Wong MCM, Chu CH, Dai L, Lo ECM. A 24-month randomized controlled trial on the success rates of restoring untreated and SDFtreated dentine caries lesions in primary teeth with the ART approach. J Dent 2020;100:103435

6. Oliveira BH, Rajendra A, Veitz-Keenan A, Niederman R. The Effect of Silver Diamine Fluoride in Preventing Caries in the Primary Dentition: A Systematic Review and MetaAnalysis. Caries Res 2019;53:24-32

7. Seifo N, Cassie H, Radford JR, Innes NPT. Silver diamine fluoride for managing carious lesions: an umbrella review. BMC Oral Health. 2019;12;145-152 
8. Chibinski AC, Wambier LM, Feltrin J, Loguercio AD, Wambier DS, Reis A. Silver Diamine Fluoride Has Efficacy in Controlling Caries Progression in Primary Teeth: A Systematic Review and Meta-Analysis. Caries Res 2017;51:527-541

9. Contreras V, Toro MJ, Elías-Boneta AR, Encarnación-Burgos A. Effectiveness of silver diamine fluoride in caries prevention and arrest: a systematic literature review. Gen Dent 2017;65:22-29

10. Agha RA, Borrelli MR, Farwana R, Koshy K, Fowler AJ, Orgill DP; PROCESS Group. The PROCESS 2018 statement: Updating Consensus Preferred Reporting Of CasE Series in Surgery (PROCESS) guidelines. Int J Surg 2018;60:279282

11. Pitts, N. "ICDAS" - an international system for caries detection and assessment being developed to facilitate caries epidemiology, research and appropriate clinical management. Community Dent Health 2004;21:193-198

12. Pitts NB, Ekstrand KR. International Caries Detection and Assessment System (ICDAS) and its International Caries Classification and Management System (ICCMS)-methods for staging of the caries process and enabling dentists to manage caries. Community Dent Oral Epidemiol 2013,41:41-52.

13. Horst JA, Ellenikiotis H, Milgrom PL. UCSF Protocol for Caries Arrest Using Silver Diamine Fluoride: Rationale, Indications and Consent. J Calif Dent Assoc 2016;44:16-28

14.Seifo N, Robertson M, MacLean J, et al. The use of silver diamine fluoride (SDF) in dental practice. Br Dent J 2020; 228:75-81

15. Horst JA. Silver Fluoride as a treatment for dental caries. Adv Dent Res 2018;29:135-140

16. Zhi QH, Lo EC, Lin HC. Randomized clinical trial on effectiveness of silver diamine fluoride and glass ionomer in arresting dentine caries in preschool children. J Dent 2012;40:962-967

17. Fung MHT, Duangthip D, Wong MCM, Lo $\mathrm{ECM}$, Chu $\mathrm{CH}$. Arresting dentine caries with different concentration and periodicity of silver diamine fluoride. JDR Clin Trans Res 2016;1:143152

18. Wakshlak RB-K, Pedahzur R, Avnir D. Antibacterial activity of silver-killed bacteria: the “zombies” effect. Sci Rep 2015;5:e9555
19. Jiang M, Mei ML, Wong MCM, Chu CH, Lo ECM. Effect of silver diamine fluoride solution application on the bond strength of dentine to adhesives and to glass ionomer cements: a systematic review. BMC Oral Health 2020;20:4050

20. Schwendicke F, Meyer-Lueckel H, Stolpe M, Dörfer CE, Paris S. Costs and effectiveness of treatment alternatives for proximal caries lesions. PLoS One 2014;9:e86992

21. Braga, M., Floriano, I., Ferreira, F.R., Silveira, J.M., Reyes, A., Tedesco et al. Are the approximal caries lesions in primary teeth a challenge to deal with? A critical appraisal of recent evidences in this field. In:Virdi MC; Emerging trends in Oral Health Sci Dent;2015:83-116

22. Hammersmith KJ, DePalo JR, Casamassimo PS, MacLean JK, Peng J. Silver diamine fluoride and fluoride varnish may halt interproximal caries progression in the primary dentition. J Clin Pediatr Dent 2020;44:7983

23. Mei ML, Zhao IS, Ito L, Lo EC, Chu CH. Prevention of secondary caries by silver diamine fluoride. Int Dent J 2016; 66:71-77

24. Felemban, O., Dabroom, W., Al-Sharif, M., Alghamdi, B., Bantan, F.S., Farie, G.A. et al. The effect of silver diamine fluoride in preventing secondary caries in primary teeth: in-vitro study. EC Dental Science 2018;17:1564-1569

25. MacLean J. Minimally invasive treatment for molar incisor hypomineralisation. Dec Dent 2018;4:18-23

26. Horst JA, Ellenikiotis H, Milgrom PL. UCSF Protocol for Caries Arrest Using Silver Diamine Fluoride: Rationale, Indications and Consent. J Calif Dent Assoc 2016;44:16-28

27. Bagher SM, Sabbagh HJ, AlJohani SM, Alharbi G, Aldajani M, Elkhodary H. Parental acceptance of the utilization of silver diamine fluoride on their child's primary and permanent teeth. Patient Prefer Adherence. 2019;13:829-835

28. Rossi G, Squassi A, Mandalunis P, Kaplan A. Effect of silver diamine fluoride (SDF) on the dentin-pulp complex: ex vivo histological analysis on human primary teeth and rat molars. Acta Odontol Latinoam. 2017;30:5-12 


\section{How to cite this article:}

Abhishek Soni, Sanchit Paul, Priyanka Sachdeva. Silver diamine fluoride as a minimally invasive and aerosol free approach in pediatric dentistry: An extensive case series. Contemp Pediatr Dent 2021:2(2):42-54. DOI: $10.51463 /$ cpd.2021.51

\section{Declarations}

Acknowledgements: Not applicable.

Conflict of Interest Statement: The authors, certify that they have no commercial associations (e.g., consultancies, stock ownership, equity interests, patent licensing arrangements, etc) that might pose a conflict of interest in connection with the submitted article

Ethics Statement: Procedure was explained to the legal guardian and written consent was obtained for the treatment and publication of the case report.

Informed Consent: Informed consent was taken from parents and/or guardians.

Author contributions: Conception and design: All Authors; Acquisition of data: AS, SP; Interpretation of data: AS, SP; Drafting article: All Authors; Revision artice: All Authors; Final approval: All Authors

Funding: This work is not finantiated.

Data Availability: The data used to support the findings of this study can be made available upon request to the corresponding author.

Peer-review: Externally double-blinded peer-reviewed. 